\title{
Seguimiento del paciente trasplantado: recomendaciones desde Atención Primaria
}

B. López Serrano, F.J . Zufia García, R. Ruiz Giardin, P. Medina Cuenca

Médicos de familia. C.S. El Espinillo. Área 11. INSALUD. Madrid

\section{RESUMEN}

El avance obtenido en el trasplante de órganos sólidos, constituye uno de los éxitos médicos más relevantes de la década pasada. El aumento de la supervivencia a largo plazo, supone la aparición de una gran variedad de complicaciones médicoquirúrgicas, la mayoría relacionadas directa o in directamente con la utilización continua de drogas inmunosupresoras, que se ha implicado como el factor determinante en estos pacientes para pre sentar mayor susceptibilidad a padecer infeccio nes, procesos malignos, hipertensión arterial, hipe ruricemia-gota, etc.

La mayoría de las enfermedades que presentan estos pacientes son similares a las del resto de la población, y pueden ser asumidas por el médico de familia; debemos por tanto conocer y saber manejar las principales complicaciones, y las activida des preventivas que debemos realizar para mejorar su supervivecia.

Palabras clave: Trasplante. Terapia inmunosu presora. Atención Primaria.
Follow up on post-transplant patients. Recommen dations from Primary Health Care

\begin{abstract}
Solid organ transplantation is one of the medical success stories of the past decade. The mark impro vement in early graft survival has resulted in more long-term successful transplant survivors, many of whom have other ongoing medical and surgical complications, and many of this complications are the direct or indirect consequences of immunosup pressive therapy, such as cardiovascular disease, secondary tumors, infections, hypertension, hype ruricemia and gout, etc.

Although medical complications are frecuent, they are usually similar to the rest of patients and easily managed. The role for primary care physi cians includes monitoring of allograft function, acute intervention of minor complications, mana gement of long term concomitant medical condi tions and preventive actions.
\end{abstract}

Key words: Transplant. Immunosuppressive the rapy. Primary Health Care.

\section{INTRODUCCIÓN}

Con los avances obtenidos durante los últimos años, el trasplante constituye la alternativa necesaria en el tratamiento de enfermedades irreversibles de distintos órganos. Tras el trasplante, los pacientes presentan problemas médicos y complicaciones frecuentes a consecuencia del tratamiento inmunosupresor que reciben: mayor susceptibilidad a pa- decer infecciones, procesos malignos, hipertensión arterial, hiperlipidemias, hiperuricemia-gota, etc. Será por tanto necesario un seguimiento a largo plazo, manteniendo una comunicación continua y fluida entre el paciente y su médico de Atención Primaria, esencial para las actividades preventivas, la detección precoz de complicaciones y por tanto asegurar el éxito del trasplante. El control evolutivo postrasplante, tradicionalmente era labor exclu- 
siva de nefrólogos, cardiólogos, digestólogos, etc. El incremento progresivo de población trasplantada y la mejoría en la supervivencia hacen necesaria la participación activa del médico de familia. Además, la mayoría de las enfermedades que presentan estos pacientes, son similares a las del resto de la población. Debemos por tanto conocer y saber manejar las principales complicaciones de estos enfermos, así como los principales efectos secundarios del tratamiento inmunosupresor que reciben. En la tabla I describimos los principales efectos secundarios que debemos conocer de las drogas inmunosupresoras, así como en la tabla II, las interacciones medicamentosas que este tipo de drogas presentan con fármacos empleados frecuentemente en la práctica médica. Nuestros conocimientos y actividades preventivas en los pacientes trasplantados, pueden contribuir a mejorar su calidad de vida y supervivencia. Analizamos a continuación las principales complicaciones médicas que pueden aparecer a largo plazo en el paciente trasplantado.

\section{INFECCIÓN POSTRASPLANTE}

A consecuencia de la terapia inmunosupresora, los pacientes trasplantados presentan mayor susceptibilidad para padecer infecciones por numero-

\section{Tabla I}

\begin{tabular}{|ll}
\hline & \multicolumn{1}{c}{ Tabla I } \\
& PRINCIPALES EFECTOS SECUNDARIOS \\
DE LAS DROGAS INMUNOSUPRESORAS
\end{tabular}

Tomada de Peddi R. 1997; 81: 767-783.
Tabla II

INTERACCIONES MEDICAMENTOSAS EN LA FARMACOCINÉTICA DE LA CICLOSPORINA ${ }^{1,6}$

Fármacos que descienden los niveles sanguíneos de ciclosporina y por tanto aumentan riesgo de rechazo Carbamacepina

Isoniacida

Rifampicina

Fenitoína

Fenobarbital

Fármacos que aumentan los niveles sanguíneos de ciclosporina y por tanto aumentan riesgo de toxicidad, sobreinmunosupresión y riesgo de infección

Claritromicina

Diltiacem

Eritromicina

Fluconazol

Itraconazol

Ketoconazol

Metoclopramida

Metilprednisolona

Nicardipino

Verapamil

Tomada de Peddi R. 1997; 81: 767-783.

sos microorganismos. Al menos el $75 \%$ de los pacientes, sufren un episodio infeccioso durante el primer año postrasplante. Las infecciones suponen la primera causa de morbi-mortalidad en el curso del postrasplante ${ }^{1}$. El riesgo de infección es determinado por la interacción entre el estado inmunosupresor del paciente y la exposición epidemiológica a la que se encuentra sometido. La mayoría de las infecciones se diagnostican en los primeros cuatro meses postrasplante:

1. Durante el primer mes las infecciones pueden ser: latentes (que se evidencian como consecuencia del estado inmunosupresor): tuberculosis, infección del tracto urinario. Transmisión de la infección desde el donante. Relacionadas con el acto quirúrgico y el postoperatorio (catéteres intravenosos, sondajes urinarios) ${ }^{1-3}$.

2. En el periodo comprendido entre el segundo y el sexto mes predominan: infecciones víricas: citomegalovirus, herpes. Infección por microorganismos intracelulares: micobacterias, listeria monoci togenes, nocardia asteroides, pneumocistis Carinii. Infecciones bacterianas secundarias a problemas técnicos en el injerto ${ }^{1-3}$.

3. A partir del sexto mes: la mayoría de los pacientes no presentan episodios infecciosos dada la buena evolución del trasplante ${ }^{1-3}$. 


\section{Prevención de la infección}

Las actividades preventivas de la infección en la etapa postrasplante incluyen:

1. Reducir la transmisión a través del órgano

2. Reducir la transmisión ambiental

3. Vacunación: en la tabla III se muestran las pautas de vacunación recomendadas.

4. Profilaxis antimicrobiana en los siguientes casos:

-Profilaxis antibiótica quirúrgica, que varía según el tipo de trasplante.

-Durante los primeros meses postrasplante, es obligada la administración de Cotrimoxazol para la prevención de la infección por pneumocistis carinii: la dosis recomendada es $800 / 160 \mathrm{mg} / 24 \mathrm{~h}$ durante un año, útil al mismo tiempo para prevenir la infección por nocardia asteroides, listeria mo nocytogenes y salmonella ${ }^{2-4}$.

—En la población seronegativa para citomegalovirus: profilaxis con Ganciclovir ${ }^{2-4}$.

-Con frecuencia se emplean antifúngicos en la prevención de infección candidiásica, aunque su uso es controvertido. Existen pocos estudios que lo avalen, por lo que actualmente no se aconseja la profilaxis universal ${ }^{2-4}$.

\section{ENFERMEDADES MALIGNAS}

La utilización de múltiples drogas inmunosupresoras, se ha implicado como el factor determinante en la mayor susceptibilidad a padecer tumores malignos en los pacientes trasplantados. La historia natural de estos tumores es usualmente más agresiva de lo que sería de esperar en pacientes no trasplantados $^{5}$. La terapia inmunosupresora crea una at- mósfera propicia para el desarrollo y crecimiento tumoral. Otros factores, como la presencia de enfermedad subyacente, efectos oncogénicos de las drogas e infecciones virales también pueden influir ${ }^{6}$. Varios tipos de tumores son más frecuentes que en la población general5:

-El carcinoma de piel es el más frecuente con una incidencia del $37 \%$, predominando el subtipo de células escamosas (a diferencia del predominio del basocelular en la población general), y su aparición es a edades más precoces, con frecuentes recurrencias y gran agresividad.

- Los linfomas se presentan con una incidencia cuatro veces superior a su incidencia en la población general. Los más frecuentes son los linfomas no Hodgkin, generalmente de células B, típicamente de localización extranodal. Su aparición se produce en torno a los 33 meses postrasplante.

- Sarcomas: existe una alta incidencia de sarcoma de Kaposi en pacientes con trasplante hepático y renal. Aparecen en torno a los 20 meses postrasplante.

- Carcinoma colorrectal: los tumores de órganos sólidos también aparecen en pacientes trasplantados con mayor incidencia de lo habitual. Su aparición es más tardía: a los 63-93 meses postransplante. En la mayoría de los casos, no se demuestra patología gastrointestinal previa, por lo que se puede asumir que son secundarios al tratamiento inmunosupresor.

La alta incidencia y la naturaleza agresiva de las neoplasias, hace necesario un estrecho control y seguimiento en los años postrasplante. Se debe incidir de un modo especial en las medidas generales de prevención: evitar tabaco y consumo de alcohol, mejorar hábitos dietéticos, etc., que son medidas simples pero efectivas. Dada la alta inci-

Tabla III

VACUNACIONES RECOMENDADAS 2

\begin{tabular}{lll}
\hline VACUNA & ORGANO SÓLIDO & MÉDULA ÓSEA \\
\hline Streptococcus Pneumoniae & $\begin{array}{c}\text { Inmunización pretrasplante } \\
\text { y revacunación cada } 5 \text { años }\end{array}$ & $\begin{array}{c}\text { Inmunización pretrasplante y } \\
\text { revacunación cada } 5 \text { años }\end{array}$ \\
Haemophilus Influenzae & Inmunización pretrasplante & Inmunización pretrasplante \\
Neisseria Meningitidis & Inmunización pretrasplante & Inmunización pretrasplante \\
Influenza & Inmunización en otoño & Inmunización en otoño \\
Sarampión-Rubeola-Parotiditis & Inmunización pretrasplante & $\begin{array}{c}\text { Inmunización a los } 24 \text { meses si no } \\
\text { aparece enf. del injerto contra huésped }\end{array}$ \\
Tétanos-Difteria & Inmunización pretrasplante. & Reinmunización postrasplante
\end{tabular}

Tomada de Pahissa Berga A 1995; LVI: 59-63. 
dencia de tumores cutáneos, se debe evitar la exposición solar, y si se produce, utilizar cremas protectoras. Siguiendo las recomendaciones de la Sociedad Americana del Cáncer, mostramos en la tabla IV los controles recomendados para el despistaje precoz de enfermedad tumoral en los pacientes trasplantados 5 .

Los marcadores tumorales no se utilizan en el screening habitual. Debemos tener en cuenta que una vigilancia exclusiva del órgano trasplantado puede ocasionar que pasen desapercibidas otras complicaciones importantes, por lo que debemos estar alerta e investigar la aparición de cualquier síntoma y toda área sospechosa debe ser biopsiada.

\section{ENFERMEDAD VASCULAR. HIPERTENSIÓN ARTERIAL}

La hipertensión arterial postrasplante aparece en más del $50 \%$ de los pacientes, requiriendo tratamiento farmacológico en la mayoría de los casos ${ }^{3}$. Afortunadamente, la mayoría responden rápida y adecuadamente al tratamiento hipotensor, pero debemos tener en cuenta que algunos agentes antihipertensivos pueden provocar complicaciones en el paciente trasplantado.
Generalmente, el tratamiento se inicia con un agente vasodilatador (ya que el principal mecanismo de producción de la hipertensión inducida por las drogas inmunosupresoras es la vasoconstricción renal: causando retención de agua y sodio). Comenzaremos con un calcioantagonista de la familia de las dihidropiridinas (nifedipino, amlodipino o felodipino). Nicardipino, diltiazem y verapamil incrementan los niveles de ciclosporina y tacrólimus por lo que deben ser evitados. Pueden emplearse bajas dosis de diuréticos, particularmente en pacientes con hipertensión sal-sensible, o si existe edema periférico. Debemos valorar que la combinación de furosemida con ciclosporina se asocia a la aparición de hiperuricemia, incrementando el riesgo de gota. Se debe tener precaución en la utilización de IECAS, al poder precipitar fallo renal en pacientes con trasplante renal con o sin estenosis de la arteria renal ${ }^{4}$.

A medida que se reduce la dosis de ciclosporina, disminuye la hipertensión arterial, y la necesidad de medicación.

La enfermedad arterioesclerótica, constituye la mayor causa de morbimortalidad tardía en pacientes trasplantados. El riesgo relativo de mortalidad por enfermedad coronaria arterial oscila del 3-10\% y en la enfermedad cerebrovascular del 1-3\% ${ }^{1}$. Los

\section{Tabla IV}

SCREENING RECOMENDADO PARA LA PREVENCIÓN DE ENFERMEDADES TUMORALES EN PACIENTES TRASPLANTADOS5

\begin{tabular}{|c|c|c|c|}
\hline TUMOR & PROCEDIMIENTO DE SCREENING & PRIMERA VALORACIÓN & INTERVALO \\
\hline Cáncer de piel & Examen físico & 3-6 meses postrasplante & Anual \\
\hline Sarcoma de Kaposi & Examen físico & 3-6 meses postrasplante & Anual \\
\hline Linfoma & $\begin{array}{l}\text { Examen físico } \\
\text { Sistemático de sangre, LDH }\end{array}$ & 3-6 meses postrasplante & Anual \\
\hline Cáncer colorrectal & Colonoscopia & $\begin{array}{l}\text { Tras trasplante en pacientes } \\
\text { con enferm. inflamatoriacon } \\
\text { intestinal. } 10 \text { años postrasplante } \\
\text { en pacientes de riesgo }\end{array}$ & $\begin{array}{l}\text { Anualmente en pacientes enf. } \\
\text { inflamatoria intestinal. } \\
\text { Cada 2-3 años en pacientes } \\
\text { de riesgo }\end{array}$ \\
\hline $\begin{array}{l}\text { Cáncer } \\
\text { hepatocelular }\end{array}$ & $\begin{array}{l}\text { Ecografía abdominal. Niveles } \\
\text { de alfafetoproteína }\end{array}$ & $\begin{array}{l}\text { 6-12 meses postrasplante } \\
\text { en pacientes con infección } \\
\text { crónica virus B }\end{array}$ & Anual \\
\hline Cáncer de cérvix & $\begin{array}{l}\text { Examen pélvico } \\
\text { Citología }\end{array}$ & $\begin{array}{l}\text { En el momento del trasplante } \\
\text { para detectar ca. in situ }\end{array}$ & $\begin{array}{l}\text { Anual. } \\
\text { Cada 2-3 años si tres } \\
\text { citologías consecutivas son } \\
\text { normales }\end{array}$ \\
\hline Cáncer de mama & $\begin{array}{l}\text { Examen físico } \\
\text { Mamografía }\end{array}$ & $\begin{array}{l}10 \text { años postrasplante } 0 \\
\text { mamografía a los } 50 \text { años }\end{array}$ & Anual \\
\hline Cáncer de próstata & $\begin{array}{l}\text { Tacto rectal } \\
\text { PSA }\end{array}$ & $\begin{array}{l}10 \text { años postrasplante o a los } \\
50 \text { años en pac. de riesgo o a } \\
\text { los } 40 \text { años en pacientes de alto } \\
\text { riesgo (historia familiar, etc.) }\end{array}$ & Anual \\
\hline
\end{tabular}

Tomada de Tan-Shalaby J 1995; 15: 156-163. 
factores que contribuyen al aumento de enfermedad vascular son: hipertensión arterial, hiperlipemia, obesidad, tabaquismo, diabetes mellitus, etc.

\section{HIPERLIPEMIA}

La hiperlipemia postrasplante es extremadamente frecuente, y contribuye al incremento de morbimortalidad por enfermedad vascular postrasplante. La hiperlipemia puede exacerbarse por el uso de corticoesteroides y ciclosporina. Se debe iniciar el manejo con un tratamiento dietético que no suele ser suficiente, por lo que en la mayoría de los casos recurriremos a la utilización de fármacos. El manejo farmacológico es controvertido debido a los efectos secundarios de la medicación. Los más efectivos y mejor tolerados son los inhibidores de la HMG-Coa reductasa, por lo que constituyen el tratamiento de elección ${ }^{4}$. Las resinas de intercambio iónico pueden disminuir la absorción de los fármacos inmunosupresores. La niacina puede producir hepatotoxicidad, hiperuricemia e hiperglucemia y el Gemfibrocil presenta como potencial efecto secundario el desarrollo de miositis y rabdomiolisis ${ }^{1,4}$.

\section{HIPERURICEMIA-GOTA}

La hiperuricemia es muy frecuente en pacientes tratados con ciclosporina y tacrólimus, alcanzando incidencias de hasta el $85 \%$ de los casos, presentándose episodios de gota en un 5-25\% de los pacientes ${ }^{7}$.

La hiperuricemia asintomática no debe ser tratada, independientemente de sus cifras. Un ataque de gota ocasional, en paciente con función renal estable, se puede tratar con antiinflamatorios no esteroideos (indometacina, ibuprofeno), que no son ne- frotóxicos en tratamientos ocasionales, o con colchicina a bajas dosis. En caso de ataques frecuentes, además del tratamiento de la crisis, el tratamiento de fondo de la hiperuricemia recomendado es el alopurinol, que interfiere con el metabolismo de la azatioprina. Por ello, la dosis de ésta se debe disminuir a un tercio de lo inicial ${ }^{7}$.

\section{HIPERCALCEMIA}

La hipercalcemia secundaria al hiperparatiroidismo es frecuente en los pacientes con fallo renal y diálisis ${ }^{7}$. La hipercalcemia suele resolverse espontáneamente en la mayoría de los pacientes tras el éxito del trasplante. Sin embargo, se produce una alta tasa de pérdida ósea secundaria al tratamiento corticoesteroideo y al hiperparatiroidismo. No existe contraindicación para recibir tratamiento con estrógenos, vitamina D, calcio, etc ${ }^{3}$.

\section{COMPLICACIONES HEMATOLÓGICAS}

1. Anemia: suele normalizarse en 90-100 días. Si persiste, puede ser debida a déficit de hierro secundario a pérdidas sanguíneas, o por supresión medular secundaria a azatioprina ${ }^{4}$.

2. Leucopenia: causada por la utilización de azatioprina.

\author{
CORRESPONDENCIA: \\ Beatriz López Serrano \\ Centro de Salud "El Espinillo" \\ Avda. de Orovilla, s/n \\ 28041 Madrid \\ Telf. 913180399
}

\section{Bibliografía}

1. Peddi R, First R. Primary care of patients with renal transplants. Medical clinics of north America 1997; 81: 767-83.

2. Pahissa Berga A. Infecciones en el paciente trasplantado: conocimientos básicos para el médico de atención primaria. Jano 1999; LVI: 59-63.

3. Pirsch JD, Friedman R. Primary care of the renal transplant patient. Journal of general internal medicine 1994; 9: 29-35.

4. Rowen K, Zetterman MD. Primary care management of the Liver Transplant patient. The American Journal of Medicine 1994; 96 (suppl 1A): 10S-17S.
5. Tan-Shalaby J, Tempero M. Malignances after liver transplantation: A comparative review. Seminars in Liver disease 1995; 15: 156-63.

6. Jonas S, Rayes N, Neumann U. De novo malignancies after liver transplantation using Tacrolimus-based protocols or Cyclosporine-based quadruple inmunosuppression with an Interleukin -2 receptor antibody or antithymocyte globulin. Cancer 1997; 80: 1141-50.

7. Grossman RA. Care of the renal transplant recipient: A field guide for the generalist. DisMon 1998; 44: 269-82. 